\title{
Eigenfunction expansions for transient diffusion in heterogeneous media
}

\author{
Carolina P. Naveira-Cotta ${ }^{\mathrm{a}}$, Renato M. Cotta ${ }^{\mathrm{a}, *}$, Helcio R.B. Orlande ${ }^{\mathrm{a}}$, Olivier Fudym ${ }^{\mathrm{a}, \mathrm{b}}$ \\ ${ }^{a}$ LTTC - Laboratory of Transmission and Technology of Heat Mechanical Engineering Department, POLI/COPPE, Universidade Federal do Rio de Janeiro, UFRJ CX. Postal 68503, \\ Cidade Universitária, 21945-970, Rio de Janeiro, RJ, Brazil \\ ${ }^{\mathrm{b}}$ RAPSODEE UMR 2392 CNRS École des Mines d'Albi, Albi, France
}

\section{A R T I C L E I N F O}

Article history:

Received 3 November 2008

Received in revised form 16 April 2009

Accepted 16 April 2009

Available online 24 June 2009

\section{Keywords:}

Heterogeneous media

Diffusion

Variable physical properties

Heat conduction

Sturm-Liouville problems

Integral transforms

\begin{abstract}
A B S T R A C T
The Generalized Integral Transform Technique (GITT) is employed in the analytical solution of transient linear heat or mass diffusion problems in heterogeneous media. The GITT is utilized to handle the associated eigenvalue problem with arbitrarily space variable coefficients, defining an eigenfunction expansion in terms of a simpler Sturm-Liouville problem of known solution. In addition, the representation of the variable coefficients as eigenfunction expansions themselves has been proposed, considerably simplifying and accelerating the integral transformation process, while permitting the analytical evaluation of the coefficients matrices that form the transformed algebraic system. The proposed methodology is challenged in solving three different classes of diffusion problems in heterogeneous media, as illustrated for the cases of thermophysical properties with large scale variations found in heat transfer analysis of functionally graded materials (FGM), of abrupt variations in multiple layer transitions and of randomly variable physical properties in dispersed systems. The convergence behavior of the proposed expansions is then critically inspected and numerical results are presented to demonstrate the applicability of the general approach and to offer a set of reference results for potentials, eigenvalues, and related quantities.
\end{abstract}

(c) 2009 Elsevier Ltd. All rights reserved.

\section{Introduction}

The analysis of diffusion processes in heterogeneous media appears in various different contexts in the physical sciences and engineering, and this terminology is rather general and frequently not sufficient to characterize a specific physical situation and its mathematical treatment. Within the context of heat conduction in heterogeneous solids, one may for instances identify a few different situations that are in general referred to in such terms, including composites with non-uniform micro-structure, multilayered composites, solids with inclusions, non-homogeneous porous materials, welded or glued surfaces, etc. The result of heterogeneity is then expressed as space variability of the related thermophysical properties, either in regular ordered form or in a stochastic functional behavior. Recently, there has been a renewed interest in the heat conduction analysis of heterogeneous solids in light of the developments on manufacturing of functionally graded materials (FGM) and nanocomposites, when the thermal properties of materials are tailored to meet requirements in specific applications, in many cases associated with extreme environments and conditions.

Diffusion problems defined in heterogeneous media involve spatially variable coefficients in different forms, depending on the type of heterogeneity involved, such as large scale variations

\footnotetext{
* Corresponding author. Tel.: +55 21256 28566; fax: +55 2125628383.

E-mail address: cotta@mecanica.coppe.ufrj.br (R.M. Cotta).
}

in functionally graded materials (FGM), abrupt variations in layered composites, and random variations due to local concentration fluctuations in dispersed phase systems such as composites, [1-4]. In all such situations, the accurate representation of the diffusion process requires a detailed local solution of the potential behavior, generally with the aid of discrete numerical solutions with sufficient mesh refinement and computational effort and/or semi-analytical approaches for specific or simplified functional forms, as discussed in [1,5-9].

Analytical solutions of linear diffusion problems have been analyzed and compiled in [10], where seven different classes of heat and mass diffusion formulations are systematically solved by the classical Integral Transform Method. The obtained formal solutions are applicable over a very broad range of problems in heat and mass transfer, in part illustrated in the referred compendium, including certain examples of diffusion in heterogeneous media. Later on, the classical approach gained a hybrid numerical-analytical implementation and was coined as the Generalized Integral Transform Technique (GITT), [11-16], offering more flexibility in handling a priori non-transformable problems, including, among others, the analysis of nonlinear diffusion and convection-diffusion problems.

The solution of the associated eigenvalue problem is the major task in the numerical computation of such formal solutions presently available, so as to provide accurate numerical values for the related eigenvalues and normalized eigenfunctions that compose the expansions inherent to the integral transform method. In a 


\section{Nomenclature}

\begin{tabular}{|c|c|}
\hline$d(\mathbf{x})$ & linear dissipation operator coefficient, Eq. (1a) \\
\hline$f(\mathbf{x})$ & initial condition, Eq. (1b) \\
\hline G & gain parameter in randomly variable properties \\
\hline$k(\mathbf{x})$ & diffusion operator coefficient, Eq. (1a) \\
\hline$M$ & $\begin{array}{l}\text { truncation order in coefficients expansion, Eqs. (9a), } \\
(10 a),(11 a)\end{array}$ \\
\hline$N$ & $\begin{array}{l}\text { truncation order in eigenvalue problem expansion, Eq. } \\
\text { (6a) }\end{array}$ \\
\hline$N_{i}$ & normalization integrals \\
\hline$P(\mathbf{x}, \mathrm{t})$ & equation source term, Eq. (1a) \\
\hline$t$ & dimensionless time variable \\
\hline$T$ & dimensionless temperature (potential) \\
\hline$u$ & transformed dependent variable, Eq. (18) \\
\hline$w(\mathbf{x})$ & transient operator coefficient, Eq. (1a) \\
\hline$x$ & dimensionless longitudinal coordinate \\
\hline$x$ & position vector \\
\hline
\end{tabular}

Greek letters:

$\alpha(\mathbf{x}), \beta(\mathbf{x})$ boundary condition coefficients, Eq. (1c)

$\beta \quad$ parameter in FGM properties variation

$\gamma \quad$ parameter in double-layer properties variation

$\delta \quad$ function in double-layer properties variation

$\phi(\mathbf{x}, t) \quad$ equation source term, Eq. (1a)

$\lambda \quad$ eigenvalue of auxiliary problem

$\mu \quad$ original problem eigenvalues

$\Omega \quad$ eigenfunction of auxiliary problem

$\psi \quad$ original problem eigenfunctions

Subscripts and superscripts

$i, n, m \quad$ order of eigenquantities

- $\quad$ integral transform

$\sim \quad$ normalized eigenfunction

number of situations, depending on the specific functional form of the equation coefficients, one may find explicit solutions for the eigenfunctions in terms of special functions which are well documented in textbooks, and more recently, readily available in symbolic computation packages [17]. On the other hand, for the more general formulation of the eigenvalue problem, a few computational approaches have been developed that offer numerical approximations of the eigenvalues and eigenfunctions, such as the Runge-Kutta method with Pruffer transformation $[18,19]$, the Sign-count method [20,21], and the GITT itself [22-24].

The GITT has been applied in the solution of eigenvalue problems to both cases of variable coefficients [22] and irregular domains [24]. The approach is here first applied in solving an example of spatially variable thermophysical properties found in heat conduction within functionally graded materials (FGM), validated by the exact solution obtained through classical integral transforms in the specific situation of exponentially varying coefficients. Then, the approach is employed for handling a double-layered system with abrupt variation of properties, and critically compared against the exact solution obtained by the classical integral transform method with the adequate multi-region eigenvalue problem for an actually discontinuous problem. The idea is to propose expansions for the desired eigenfunctions themselves, based on an auxiliary problem of known solution. Then, the integral transformation process as applied to the original Sturm-Liouville problem, yields an algebraic transformed system of equations, written as a matrix eigensystem analysis. The numerically computed matrix eigenvalues and eigenvectors allow the reconstruction of the original problem eigenfunctions, which are actually needed in the analytical solution of the proposed diffusion problem.

The present work also considers the possibility of expressing the variable coefficients themselves as eigenfunction expansions, not necessarily of the same auxiliary eigenvalue problem. This is particularly advantageous in the evaluation of the algebraic system coefficients matrices, which result from the integration transformation process. All the related integrals can then be expressed in terms of simple eigenfunctions, in general allowing for straightforward analytical evaluations, instead of costly numerical integrations, especially for multidimensional applications. The present methodology can thus be particularly suitable in properties identification tasks and optimization for material properties tailoring. Thus, the approach employing the expanded coefficients is here challenged to handle the same double-layer abrupt variation situation already considered, and then demonstrated for a situation of intense random variation of the equation coefficients along the space variable, such as in [1]. After demonstrating the convergence behavior of the related eigenquantities, the random variation case is critically examined against the approximation of effective thermophysical properties values for a range of amplitudes in the maximum variation allowed for.

\section{Analysis}

We consider a general formulation on transient diffusion for the potential $T(\mathbf{x}, t)$ dependent on position $\mathbf{x}$ and time $t$ and defined in region $V$ with boundary surface $S$. The formulation includes the transient term, the diffusion operator, a linear dissipation term, and an independent source term $[10,12]$, as shown in problem (1) below. The coefficients $w(\mathbf{x}), k(\mathbf{x})$, and $d(\mathbf{x})$, are responsible for the information related to the heterogeneity of the medium. The diffusion equation and initial and boundary conditions are given by:

$$
\begin{aligned}
& w(\mathbf{x}) \frac{\partial T(\mathbf{x}, t)}{\partial t}=\nabla \cdot k(\mathbf{x}) \nabla T(\mathbf{x}, t)-d(\mathbf{x}) T(\mathbf{x}, t)+P(\mathbf{x}, t), \mathbf{x} \in V, t>0 \\
& T(\mathbf{x}, 0)=f(\mathbf{x}), \quad \mathbf{x} \in V \\
& \alpha(\mathbf{x}) T(\mathbf{x}, t)+\beta(\mathbf{x}) k(\mathbf{x}) \frac{\partial T(\mathbf{x}, t)}{\partial \mathbf{n}}=\phi(\mathbf{x}, t), \quad \mathbf{x} \in S
\end{aligned}
$$

The exact solution of problem (1) is obtained with the classical integral transform method [10], and is written as:

$T(\mathbf{x}, t)=\sum_{i=1}^{\infty} \tilde{\Psi}_{i}(\mathbf{x})\left(\bar{f}_{i} e^{-\mu_{i}^{2} t}+\int_{0}^{t} \bar{g}_{i}\left(t^{\prime}\right) e^{-\mu_{i}^{2}\left(t-t^{\prime}\right)} d t^{\prime}\right)$

where the eigenvalues $\mu_{i}$ and eigenfunctions $\psi_{i}(\mathbf{x})$, are obtained from the eigenvalue problem that contains the information about the heterogeneous medium, in the form:

$\nabla \cdot k(\mathbf{x}) \nabla \psi_{i}(\mathbf{x})+\left(\mu_{i}^{2} w(\mathbf{x})-d(\mathbf{x})\right) \psi_{i}(\mathbf{x})=0, \mathbf{x} \in V$

with boundary conditions

$\alpha(\mathbf{x}) \psi_{i}(\mathbf{x})+\beta(\mathbf{x}) k(\mathbf{x}) \frac{\partial \psi_{i}(\mathbf{x})}{\partial \mathbf{n}}=0, \quad \mathbf{x} \in S$

Also, the other quantities that appear in the exact solution (2) are computed after solving problem (3), such as:

$N_{i}=\int_{V} w(\mathbf{x}) \psi_{i}^{2}(\mathbf{x}) d v, \quad$ normalization integrals

$\tilde{\psi}_{i}(\mathbf{x})=\frac{\psi_{i}(\mathbf{x})}{\sqrt{N_{i}}}, \quad$ normalized eigenfunctions

$\bar{f}_{i}=\int_{V} w(\mathbf{x}) \tilde{\psi}_{i}(\mathbf{x}) f(\mathbf{x}) d v, \quad$ transformed initial condition 
$\bar{g}_{i}(t)=\int_{V} P(\mathbf{x}, t) \tilde{\psi}_{i}(\mathbf{x}) d v+\int_{S} \phi(\mathbf{x}, t)\left[\frac{\tilde{\psi}_{i}(\mathbf{x})-k(\mathbf{x}) \frac{\partial \tilde{\psi}_{i}(\mathbf{x})}{\partial \mathbf{n}}}{\alpha(\mathbf{x})+\beta(\mathbf{x})}\right] d s$,

source terms

For a general purpose automatic implementation, it is quite desirable to develop a flexible computational approach to handle eigenvalue problems with arbitrarily variable coefficients, such as problem (3). Thus, the Generalized Integral Transform Technique (GITT) is here employed in the solution of the Sturm-Liouville problem (3) via the proposition of a simpler auxiliary eigenvalue problem, and expanding the unknown eigenfunctions in terms of the chosen basis. Also, the variable equation coefficients are themselves expanded in terms of known eigenfunctions, so as to allow for a fully analytical implementation of the coefficients matrices in the transformed system. The solution of problem (3) is thus proposed as an eigenfunction expansion, in terms of a simpler auxiliary eigenvalue problem, given as:

$\nabla \cdot k^{*}(\mathbf{x}) \nabla \Omega_{n}(\mathbf{x})+\left(\lambda_{n}^{2} w^{*}(\mathbf{x})-d^{*}(\mathbf{x})\right) \Omega_{n}(\mathbf{x})=0, \quad \mathbf{x} \in V$

with boundary conditions

$\alpha^{*}(\mathbf{x}) \Omega_{n}(\mathbf{x})+\beta^{*}(\mathbf{x}) k^{*}(\mathbf{x}) \frac{\partial \Omega_{n}(\mathbf{x})}{\partial \mathbf{n}}=0, \quad \mathbf{x} \in S$

where the coefficients, $w^{*}(\mathbf{x}), k^{*}(\mathbf{x})$, and $d^{*}(\mathbf{x})$, are simpler forms of the equation coefficients chosen so as to allow for an analytical solution of the auxiliary problem. Thus, the solution of problem (5), which needs to be known in terms of the eigenfunctions $\Omega_{n}(\mathbf{x})$ and related eigenvalues $\lambda_{n}$, offers a basis itself for the eigenfunction expansion of the original eigenvalue problem (3). Also, even the boundary conditions types of the original and auxiliary problems are allowed to be different, in case further simplification of the auxiliary function is desired, in light of the different boundary condition coefficients, $\alpha^{*}(\mathbf{x})$ and $\beta^{*}(\mathbf{x})$. The eigenfunctions $\Omega_{n}(\mathbf{x})$, may be for instance derived via separation of variables for a general multidimensional formulation, and the separated problems directly obtained from tabulated solutions of classical eigenvalue problems or computed from the ordinary differential equations via modern symbolic platforms, such as [17].

Once the eigenfunctions $\Omega_{n}(\mathbf{x})$ have been analytically constructed and the eigenvalues $\lambda_{n}$ have been computed, the proposed expansion of the original expansion is then given by:

$\Psi_{i}(\mathbf{x})=\sum_{n=1}^{\infty} \widetilde{\Omega}_{n}(\mathbf{x}) \bar{\Psi}_{i, n}, \quad$ inverse

$\bar{\Psi}_{i, n}=\int_{V} w^{*}(\mathbf{x}) \Psi_{i}(\mathbf{x}) \widetilde{\Omega}_{n}(\mathbf{x}) d v, \quad$ transform

The integral transformation is thus performed by operating Eq (3a) on with $\int_{V} \widetilde{\Omega}_{n}(\mathbf{x})-d v$. After employing Green's 2 nd formula so as to account for the difference in boundary conditions of the two eigenvalue problems, it results:

$\int_{V} \Psi_{i}(\mathbf{x})\left(\nabla \cdot k(\mathbf{x}) \nabla \tilde{\Omega}_{n}(\mathbf{x})\right) d v+\int_{S} k(\mathbf{x})\left(\Psi_{i}(\mathbf{x}) \frac{\partial \tilde{\Omega}_{n}(\mathbf{x})}{\partial \mathbf{n}}-\tilde{\Omega}_{n}(\mathbf{x}) \frac{\partial \Psi_{i}(\mathbf{x})}{\partial \mathbf{n}}\right) d s$

$$
+\int_{V} \tilde{\Omega}_{n}(\mathbf{x})\left(\mu_{i}^{2} w(\mathbf{x})-d(\mathbf{x})\right) \Psi_{i}(\mathbf{x}) d v=0
$$

Now, by combining boundary conditions ( $3 b$ ) and (5b), the surface integral above can be written as:

$$
\begin{array}{rl}
\int_{S} & k(\mathbf{x})\left(\Psi_{i}(\mathbf{x}) \frac{\partial \tilde{\Omega}_{n}(\mathbf{x})}{\partial \mathbf{n}}-\tilde{\Omega}_{n}(\mathbf{x}) \frac{\partial \Psi_{i}(\mathbf{x})}{\partial \mathbf{n}}\right) d s \\
= & \int_{S} k(\mathbf{x})\left(\frac{\alpha(\mathbf{x})}{\beta(\mathbf{x}) k(\mathbf{x})}-\frac{\alpha^{*}(\mathbf{x})}{\beta^{*}(\mathbf{x}) k^{*}(\mathbf{x})}\right)\left(\Psi_{i}(\mathbf{x}) \tilde{\Omega}_{n}(\mathbf{x})\right) d s \\
= & \int_{S} k(\mathbf{x})\left(\frac{\beta^{*}(\mathbf{x}) k^{*}(\mathbf{x})}{\alpha^{*}(\mathbf{x})}-\frac{\beta(\mathbf{x}) k(\mathbf{x})}{\alpha(\mathbf{x})}\right)\left(\frac{\partial \Psi_{i}(\mathbf{x})}{\partial \mathbf{n}} \frac{\partial \tilde{\Omega}_{n}(\mathbf{x})}{\partial \mathbf{n}}\right) d s \\
= & -\int_{S} k(\mathbf{x})\left(\frac{\beta^{*}(\mathbf{x}) k^{*}(\mathbf{x}) \alpha(\mathbf{x})}{\beta(\mathbf{x}) k(\mathbf{x}) \alpha^{*}(\mathbf{x})}-1\right)\left(\Psi_{i}(\mathbf{x}) \frac{\partial \tilde{\Omega}_{n}(\mathbf{x})}{\partial \mathbf{n}}\right) d s
\end{array}
$$

and Eq. (7a) can be for instance rewritten as:

$$
\begin{aligned}
& \int_{V} \Psi_{i}(\mathbf{x})\left(\nabla \cdot k(\mathbf{x}) \nabla \tilde{\Omega}_{n}(\mathbf{x})\right) d v \\
& \quad+\int_{S} k(\mathbf{x})\left(1-\frac{\beta^{*}(\mathbf{x}) k^{*}(\mathbf{x}) \alpha(\mathbf{x})}{\beta(\mathbf{x}) k(\mathbf{x}) \alpha^{*}(\mathbf{x})}\right)\left(\Psi_{i}(\mathbf{x}) \frac{\partial \tilde{\Omega}_{n}(\mathbf{x})}{\partial \mathbf{n}}\right) d s \\
& \quad+\int_{V} \tilde{\Omega}_{n}(\mathbf{x})\left(\mu_{i}^{2} w(\mathbf{x})-d(\mathbf{x})\right) \Psi_{i}(\mathbf{x}) d v=0
\end{aligned}
$$

Substitution of the inverse formula yields the following algebraic problem:

$$
\begin{aligned}
& \sum_{m=1}^{\infty} \bar{\Psi}_{i, m}\left(\int_{V} \tilde{\Omega}_{m}(\mathbf{x})\left(\nabla \cdot k(\mathbf{x}) \nabla \tilde{\Omega}_{n}(\mathbf{x})\right) d v\right. \\
& \quad+\int_{S} k(\mathbf{x})\left(1-\frac{\beta^{*}(\mathbf{x}) k^{*}(\mathbf{x}) \alpha(\mathbf{x})}{\beta(\mathbf{x}) k(\mathbf{x}) \alpha^{*}(\mathbf{x})}\right)\left(\tilde{\Omega}_{m}(\mathbf{x}) \frac{\partial \tilde{\Omega}_{n}(\mathbf{x})}{\partial \mathbf{n}}\right) d s \\
& \left.\quad+\int_{V} \tilde{\Omega}_{n}(\mathbf{x})\left(\mu_{i}^{2} w(\mathbf{x})-d(\mathbf{x})\right) \tilde{\Omega}_{m}(\mathbf{x}) d v\right)=0
\end{aligned}
$$

In matrix form, the eigensystem is concisely given by:

$\left(\mathbf{A}-\mu^{2} \mathbf{B}\right) \overline{\boldsymbol{\Psi}}=0$

$$
\overline{\mathbf{\Psi}}=\left\{\overline{\boldsymbol{\psi}}_{n, m}\right\} ; \quad \mathbf{B}=\left\{B_{n, m}\right\}, \quad B_{n, m}=\int_{V} w(\mathbf{x}) \tilde{\Omega}_{n}(\mathbf{x}) r \tilde{\Omega}_{m}(\mathbf{x}) d v
$$

$$
\begin{aligned}
\mathbf{A}=\{ & \left.A_{n, m}\right\} \\
A_{n, m}= & \int_{V} \tilde{\Omega}_{m}(\mathbf{x})\left(\nabla \cdot k(\mathbf{x}) \nabla \tilde{\Omega}_{n}(\mathbf{x})\right) d v \\
& +\int_{S} k(\mathbf{x})\left(1-\frac{\beta^{*}(\mathbf{x}) k^{*}(\mathbf{x}) \alpha(\mathbf{x})}{\beta(\mathbf{x}) k(\mathbf{x}) \alpha^{*}(\mathbf{x})}\right)\left(\tilde{\Omega}_{m}(\mathbf{x}) \frac{\partial \tilde{\Omega}_{n}(\mathbf{x})}{\partial \mathbf{n}}\right) d s \\
& -\int_{V} d(\mathbf{x}) \tilde{\Omega}_{n}(\mathbf{x}) \tilde{\Omega}_{m}(\mathbf{x}) d v
\end{aligned}
$$

Also, accounting for the auxiliary problem formulation, the system matrix $\mathbf{A}$ can be rewritten as:

$$
\begin{aligned}
A_{n, m}= & \int_{V} \tilde{\Omega}_{m}(\mathbf{x})\left(\nabla \cdot\left(k(\mathbf{x})-k^{*}(\mathbf{x})\right) \nabla \tilde{\Omega}_{n}(\mathbf{x})\right) d v \\
& +\int_{S}\left(k(\mathbf{x})-\frac{\beta^{*}(\mathbf{x}) \alpha(\mathbf{x})}{\beta(\mathbf{x}) \alpha^{*}(\mathbf{x})} k^{*}(\mathbf{x})\right)\left(\tilde{\Omega}_{m}(\mathbf{x}) \frac{\partial \tilde{\Omega}_{n}(\mathbf{x})}{\partial \mathbf{n}}\right) d s \\
& -\int_{V}\left(d(\mathbf{x})-d^{*}(\mathbf{x})\right) \tilde{\Omega}_{n}(\mathbf{x}) \tilde{\Omega}_{m}(\mathbf{x}) d v+\lambda_{n}^{2} \delta_{n, m}
\end{aligned}
$$

The algebraic problem (8) can be numerically solved to provide results for the eigenvalues $\mu^{2}$ and eigenvectors $\overline{\boldsymbol{\Psi}}$ from this matrix eigenvalue problem analysis [17], which will be combined by the inverse formula (6a) to provide the desired eigenfunctions.

It is also relevant to consider the possibility of expressing the variable coefficients themselves as eigenfunction expansions, in general not of the same auxiliary eigenvalue problem. This is particularly advantageous in the evaluation of the algebraic system coefficients, $A_{n, m}$ and $B_{n, m}$. All the related integrals can then be expressed in terms of eigenfunctions, in general allowing for straightforward analytical evaluations. For instance, the coefficient $w(\mathbf{x})$ can be expanded in terms of eigenfunctions, together with a filtering solution to enhance convergence, in the following form:

$$
\begin{aligned}
& w(\mathbf{x})=w_{f}(\mathbf{x})+\sum_{k=1}^{\infty} \tilde{\Gamma}_{k}(\mathbf{x}) \bar{w}_{k}, \quad \text { inverse } \\
& \bar{w}_{k}=\int_{V} \hat{w}(\mathbf{x})\left[w(\mathbf{x})-w_{f}(\mathbf{x})\right] \tilde{\Gamma}_{k}(\mathbf{x}) d \mathbf{x}, \quad \text { transform }
\end{aligned}
$$

where $\hat{w}(\mathbf{x})$ is the weighting function for the chosen normalized eigenfunction $\tilde{\Gamma}_{k}(\mathbf{x})$. For instance, the eigenfunction basis may be chosen employing the same auxiliary problem equation, but with 
first order boundary conditions throughout, while the filtering function would be a simple analytic function that satisfies the boundary values for the original coefficients. Then, once the transformed coefficients have been obtained through the transform formula, Eq. (9b), computations may be carried on with the inverse expression for the variable coefficient, Eq. (9a). This procedure might also be of interest in function estimation tasks, when the transformed quantities would be the parameters to be estimated. The two remaining coefficients are equally expanded, if necessary, in terms of eigenfunctions, here assumed to be equal just for the sake of conciseness, to yield:

$k(\mathbf{x})=k_{f}(\mathbf{x})+\sum_{k=1}^{\infty} \tilde{\Gamma}_{k}(\mathbf{x}) \bar{k}_{k}, \quad$ inverse

$\bar{k}_{k}=\int_{V} \hat{w}(\mathbf{x})\left[k(\mathbf{x})-k_{f}(\mathbf{x})\right] \tilde{\Gamma}_{k}(\mathbf{x}) d \mathbf{x}, \quad$ transform

$d(\mathbf{x})=d_{f}(\mathbf{x})+\sum_{k=1}^{\infty} \tilde{\Gamma}_{k}(\mathbf{x}) \bar{d}_{k}, \quad$ inverse

$\bar{d}_{k}=\int_{V} \hat{w}(\mathbf{x})\left[d(\mathbf{x})-d_{f}(\mathbf{x})\right] \tilde{\Gamma}_{k}(\mathbf{x}) d \mathbf{x}, \quad$ transform

The matrices coefficients may then be rewritten in terms of the expanded functions, such as for the elements of matrix $\mathbf{B}$ :

$B_{n, m}=\int_{V} w_{f}(\mathbf{x}) \tilde{\Omega}_{n}(\mathbf{x}) \tilde{\Omega}_{m}(\mathbf{x}) d v+\sum_{k=1}^{\infty} \bar{w}_{k} \int_{V} \tilde{\Gamma}_{k}(\mathbf{x}) \tilde{\Omega}_{n}(\mathbf{x}) \tilde{\Omega}_{m}(\mathbf{x}) d v$

and for matrix $\mathbf{A}$ :

$$
\begin{aligned}
A_{n, m}= & \int_{V} \tilde{\Omega}_{m}(\mathbf{x})\left(\nabla \cdot\left(k_{f}(\mathbf{x})-k^{*}(\mathbf{x})\right) \nabla \tilde{\Omega}_{n}(\mathbf{x})\right) d v \\
& +\sum_{k=1}^{\infty}\left[\int_{V} \tilde{\Omega}_{m}(\mathbf{x})\left(\nabla \cdot \tilde{\Gamma}_{k}(\mathbf{x}) \nabla \tilde{\Omega}_{n}(\mathbf{x})\right) d v\right] \bar{k}_{k} \\
& +\int_{S}\left[k_{f}(\mathbf{x})-\frac{\beta^{*}(\mathbf{x}) \alpha(\mathbf{x})}{\beta(\mathbf{x}) \alpha^{*}(\mathbf{x})} k^{*}(\mathbf{x})\right]\left(\tilde{\Omega}_{m}(\mathbf{x}) \frac{\partial \tilde{\Omega}_{n}(\mathbf{x})}{\partial \mathbf{n}}\right) d s \\
& +\sum_{k=1}^{\infty}\left[\int_{S} \tilde{\Gamma}_{k}(\mathbf{x})\left(\tilde{\Omega}_{m}(\mathbf{x}) \frac{\partial \tilde{\Omega}_{n}(\mathbf{x})}{\partial \mathbf{n}}\right) d s\right] \bar{k}_{k} \\
& -\int_{V}\left(d_{f}(\mathbf{x})-d^{*}(\mathbf{x})\right) \tilde{\Omega}_{n}(\mathbf{x}) \tilde{\Omega}_{m}(\mathbf{x}) d v \\
& -\sum_{k=1}^{\infty}\left[\int_{V} \tilde{\Gamma}_{k}(\mathbf{x}) \tilde{\Omega}_{n}(\mathbf{x}) \tilde{\Omega}_{m}(\mathbf{x}) d v\right] \bar{d}_{k}+\lambda_{n}^{2} \delta_{n, m}
\end{aligned}
$$

The norms are then computed from:

$$
\begin{aligned}
N_{i}= & \sum_{n=1}^{\infty} \sum_{m=1}^{\infty} \bar{\psi}_{i, n} \bar{\psi}_{i, m}\left\{\int_{V} w_{f}(\mathbf{x}) \tilde{\Omega}_{n}(\mathbf{x}) \tilde{\Omega}_{m}(\mathbf{x}) d v\right. \\
& \left.+\sum_{k=1}^{\infty}\left[\int_{V} \tilde{\Gamma}_{k}(\mathbf{x}) \tilde{\Omega}_{n}(\mathbf{x}) \tilde{\Omega}_{m}(\mathbf{x}) d v\right] \bar{w}_{k}\right\}
\end{aligned}
$$

\section{Applications}

The problems here considered involve the analysis of three quite different situations, first, an example of variable coefficients with large scale changes is drawn from the literature related to the heat transfer analysis of functionally graded materials (FGM) [7], another one related with an abrupt variation of thermophysical properties, typical of the transition between two different materials layers [25], and the third associated with random variation of the thermophysical properties [1].

The related dimensionless energy equation and initial and boundary conditions for the FGM example are written as [7]:

$w(x) \frac{\partial T(x, t)}{\partial t}=\frac{\partial}{\partial x}\left[k(x) \frac{\partial T(x, t)}{\partial x}\right], 0<x<1, \quad t>0$ with initial and boundary conditions

$T(x, 0)=f(x), \quad 0<x<1$

$T(0, t)=0, \quad T(1, t)=0, \quad t>0$

where the thermophysical properties are assumed to vary exponentially in the form [7]:

$k(x)=k_{0} e^{2 \beta x}, \quad w(x)=w_{0} e^{2 \beta x}, \quad \alpha_{0}=\frac{k_{0}}{w_{0}}=$ const.

$(15 a-c)$

This particular choice of functional forms leads to a problem formulation that allows for an exact solution via the classical integral transform method [10], yielding a benchmark solution for the variable coefficients case. Thus, after manipulating the coefficients within Eq. (14a), one finds:

$\frac{1}{\alpha_{0}} \frac{\partial T(x, t)}{\partial t}=\frac{\partial^{2} T(x, t)}{\partial x^{2}}+2 \beta \frac{\partial T(x, t)}{\partial x}, 0<x<1, \quad t>0$

In addition, a dependent variable transformation can recover the usual heat conduction equation form, as:

$T(x, t)=u(x, t) e^{-\beta\left(x+\beta \alpha_{0} t\right)}$

Then, the transformed problem becomes:

$\frac{1}{\alpha_{0}} \frac{\partial u(x, t)}{\partial t}=\frac{\partial^{2} u(x, t)}{\partial x^{2}}, 0<x<1, \quad t>0$

with initial and boundary conditions

$u(x, 0)=f^{*}(x)=f(x) e^{\beta x}, \quad 0<x<1$

$u(0, t)=0, \quad u(1, t)=0, \quad t>0$

The first application was solved for different values of the parameter $\beta$, with the initial condition given by:

$f(x)=\frac{1-e^{2 \beta(1-x)}}{1-e^{2 \beta}}$

which corresponds to the steady-state solution for the case of prescribed temperatures $T(0, t)=1$ and $T(1, t)=0$. Problem (18) can then be directly solved in analytical form for verification purposes, employing the classical integral transform method.

The problem formulation for the other two cases is given by:

$w(x) \frac{\partial T(x, t)}{\partial t}=\frac{\partial}{\partial x}\left[k(x) \frac{\partial T(x, t)}{\partial x}\right], 0<x<1, \quad t>0$

with initial and boundary conditions

$T(x, 0)=f(x), \quad 0<x<1$

$\left.\frac{\partial T(x, t)}{\partial x}\right|_{x=0}=0,\left.\quad \frac{\partial T(x, t)}{\partial x}\right|_{x=1}=0, \quad t>0$

The initial condition is arbitrarily chosen as $f(x)=1-x^{2}$ for the present illustration. The space variable coefficients for the abrupt variation are governed by the parameter $\gamma$ in the function below: $k(x)=k_{1}+\left(k_{2}-k_{1}\right) \delta(x), \quad w(x)=w_{1}+\left(w_{2}-w_{1}\right) \delta(x)$

$\delta(x)=\frac{1}{1+e^{-\gamma(x-x c)}}$

with $x_{c}$ being the interface position.

The randomly generated coefficients were obtained based on the example of [1], by first generating a number of positions within the medium, and then producing random scaling factors for the properties variations at each position, normalized by their average value. The resulting values are then interpolated to provide continuous functions. A gain parameter is also defined to allow for an inspection of the accuracy of effective thermophysical properties 
in simplifying the problem formulation, as shall be discussed in the results and discussion section. The variable coefficients are the given by:

$k(x)=k_{0}\left[1+\left(\frac{g_{1}(x)}{\bar{g}_{1}}-1\right) G\right], \quad w(x)=w_{0}\left[1+\left(\frac{g_{2}(x)}{\bar{g}_{2}}-1\right) G\right]$

where $g_{1}(x)$ and $g_{2}(x)$ are linearly interpolated functions within selected positions $x$ and properties values randomly generated in the interval $[0,1]$. Here, just for illustration purposes, the $x$ positions were taken as equally distributed points within the domain. For $G=1$ one obtains the full random pattern of the generated functions, while $G=0$ recovers the uniform thermophysical properties situation.

The eigenvalue problem to be solved is then given by:

$\frac{d}{d x}\left[k(x) \frac{d \psi_{i}(x)}{d x}\right]+\mu_{i}^{2} w(x) \psi_{i}(x)=0, \quad 0<x<1$

with boundary conditions

$\left.\frac{d \psi_{i}(x)}{d x}\right|_{x=0}=0,\left.\quad \frac{d \psi_{i}(x)}{d x}\right|_{x=1}=0$

So as to demonstrate the potential applicability of the present approach, the simplest possible auxiliary problem was considered, based on the choice of coefficients $k^{*}(x)=1, w^{*}(x)=1$, and $d^{*}(x)=0$, and maintaining the same boundary conditions as in Eqs. (23b,c), which results in:

$\tilde{\Omega}_{n}(x)=\sqrt{2} \cos \left(\lambda_{n} x\right)$, and $\tilde{\Omega}_{0}(x)=1$, with $\lambda_{n}=n \pi, \quad n=0,1,2 \ldots$

$(24 a-c)$

The resulting algebraic problem (8) is then numerically solved to provide results for the eigenvalues $\mu^{2}$ and eigenvectors $\overline{\mathbf{\Psi}}$, making use of the Mathematica system [17].

\section{Results and discussion}

The proposed approach was implemented in the mixed symbolic-numerical platform Mathematica 5.2 [17] and a few representative results were obtained to illustrate the convergence behavior of the eigenfunction expansions for the original eigenvalue problem with variable coefficients and the finally obtained temperature fields. The constructed notebook also offers the possibility of expanding the equation coefficients themselves in terms of eigenfunctions, as shall be examined in the results that follow.

Fig. 1 below illustrates the effect of the parameter $\beta$ on the behavior of the thermophysical properties for the first example, related to a marked variation of thermophysical properties such as in FGM thermal interfaces. Note that for the case of $\beta=3$, a ratio of approximately 400 times is achieved between the $k(x)$ values at the two edges of the domain.

Numerical results for eigenvalues and temperature distributions in the FGM example are reported below, for the numerical values of $\beta=-3,-1,1$, and 3 , and with $w_{0}=10$ and $k_{0}=1$. The equation coefficients are taken in their original analytical forms, without expanding them in eigenfunctions at the present illustration. First, Tables $1 \mathrm{a}$ and $\mathrm{b}$ illustrate the excellent convergence behavior of the first 10 eigenvalues associated with the original problem, Eqs. (14), with space variable coefficients, $k(x)$ and $w(x)$, as in Eqs. (15a,b). The different columns correspond to increasing truncation orders in the expansion of the original eigenfunctions in terms of the auxiliary problem eigenfunctions, with $N=20,30$, 40 , and 50 . It should be noticed that the first 10 eigenvalues are fully converged to the sixth significant digit in the case of $\beta=1$ (Table 1a), to within 50 terms in the eigenfunction expansion,

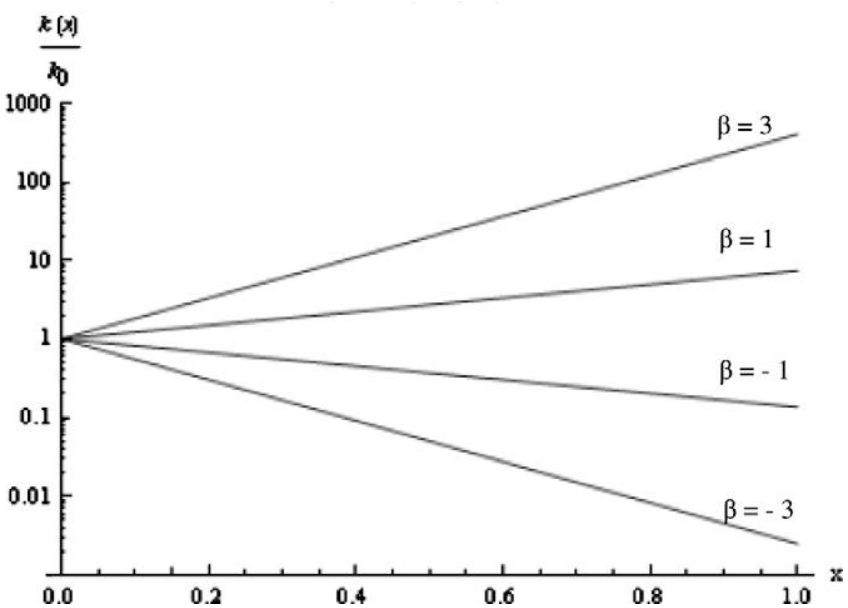

Fig. 1. Behavior of the variable diffusion coefficient $k(x)$ for the FGM example, with $\beta=-3,-1,1$, and 3 .

Table la

Convergence of eigenvalues for FGM problem, Eq. (14), with $\beta=1$.

\begin{tabular}{lllll}
\hline Eigenvalue $\mu_{i}$ & $N=20$ & $N=30$ & $N=40$ & $N=50$ \\
\hline 1 & 1.04258 & 1.04258 & 1.04257 & 1.04257 \\
2 & 2.01194 & 2.01193 & 2.01193 & 2.01193 \\
3 & 2.99712 & 2.99711 & 2.99711 & 2.99711 \\
4 & 3.98643 & 3.98641 & 3.98640 & 3.98640 \\
5 & 4.97738 & 4.97736 & 4.97735 & 4.97735 \\
6 & 5.96918 & 5.96915 & 5.96914 & 5.96914 \\
7 & 6.96145 & 6.96141 & 6.96140 & 6.96140 \\
8 & 7.95403 & 7.95398 & 7.95397 & 7.95396 \\
9 & 8.94680 & 8.94674 & 8.94673 & 8.94672 \\
10 & 9.93973 & 9.93964 & 9.93963 & 9.93962 \\
\hline
\end{tabular}

Table 1b

Convergence of eigenvalues for FGM problem, Eq. (14), with $\beta=3$.

\begin{tabular}{lllll}
\hline Eigenvalue $\mu_{i}$ & $N=20$ & $N=30$ & $N=40$ & $N=50$ \\
\hline 1 & 1.37371 & 1.37368 & 1.37367 & 1.37367 \\
2 & 2.20190 & 2.20182 & 2.20180 & 2.20179 \\
3 & 3.12789 & 3.12777 & 3.12774 & 3.12773 \\
4 & 4.08578 & 4.08558 & 4.08554 & 4.08552 \\
5 & 5.05739 & 5.05716 & 5.05711 & 5.05709 \\
6 & 6.03623 & 6.03589 & 6.03582 & 6.03580 \\
7 & 7.01911 & 7.01875 & 7.01868 & 7.01865 \\
8 & 8.00481 & 8.00426 & 8.00416 & 8.00412 \\
9 & 8.99207 & 8.99150 & 8.99139 & 8.99135 \\
10 & 9.98090 & 9.98001 & 9.97987 & 9.97982 \\
\hline
\end{tabular}

while five digits are fully converged for all the eigenvalues in the second situation, $\beta=3$ (Table $1 \mathrm{~b}$ ).

Fig. $2 \mathrm{a}$ and $\mathrm{b}$ illustrate the transient behavior of the temperature profiles for three time values, $t=0.01,0.05$, and 0.1 , for the two extreme situations considered with $\beta=3$ and -3 , respectively. In the first case, the thermophysical properties increase approximately 400 times in the direction of the lowest values of temperature, i.e. the right hand side of the graph, where both the thermal conductivity and capacity are markedly augmented, and the cooling effect is very effective. In the second case, the thermophysical properties are markedly reduced towards the edge $x=1$, sensibly affecting the cooling of the slab. It should be recalled that the dimensionless thermal diffusivity $\left(\alpha_{0}\right)$ is kept the same in both examples, but the initial conditions are different in light of the variation in the $\beta$ values. In addition, plotted with symbols are the exact results for the specific functional form of the coefficients here 

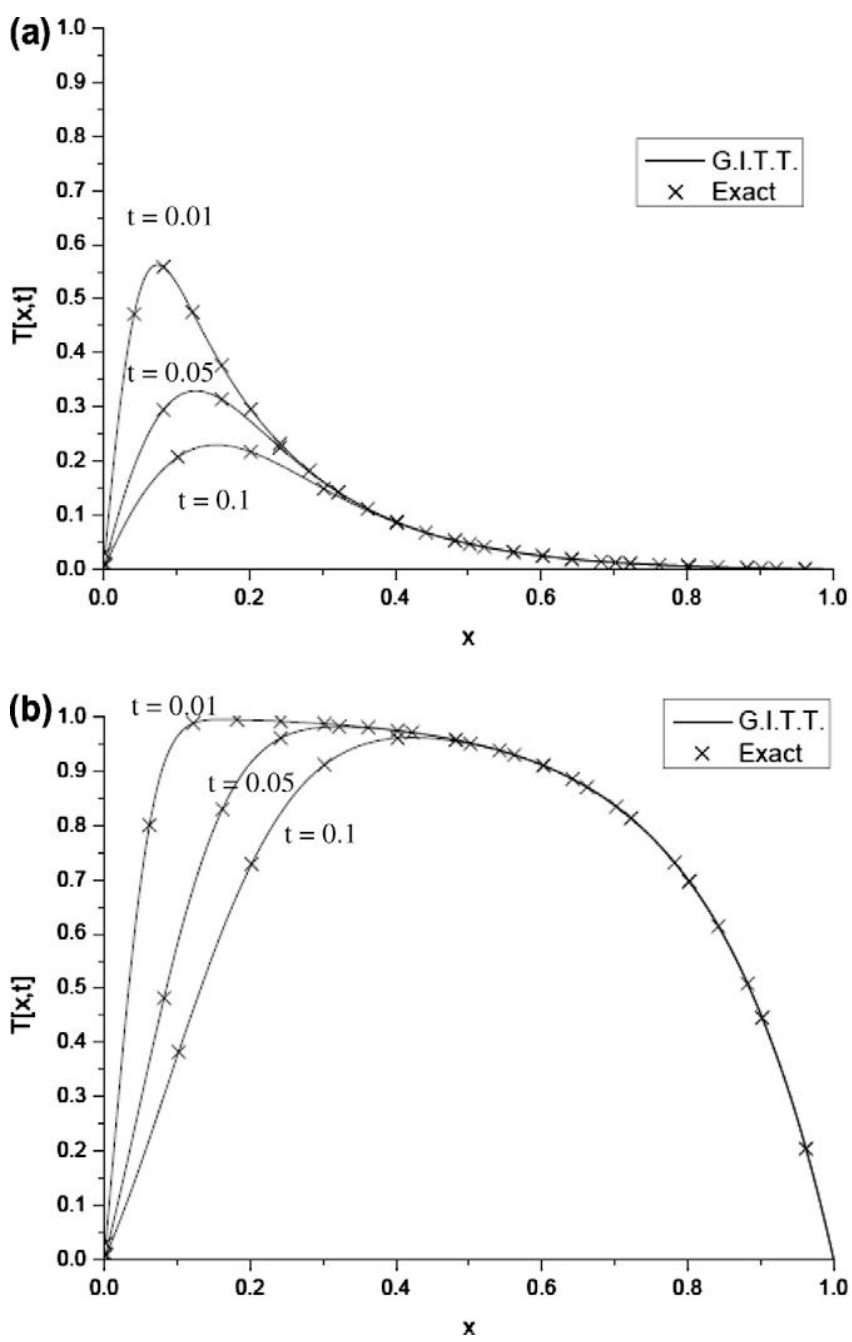

Fig. 2. (a) Physical behavior and validation (GITT $\times$ Exact) of temperature distributions for the FGM problem $(\beta=3)$. (b) Physical behavior and validation $($ GITT $\times$ Exact $)$ of temperature distributions for the FGM problem $(\beta=-3)$.

considered, as obtained from the solution of Eq. (18), for validation purposes of both sets of curves, with a perfect agreement against the GITT results here reported with 50 terms in the eigenfunction expansions.

Fig. 3 illustrates the behavior of the variable coefficient $k(x)$ for the double-layer example with a transition region, with $k_{1}=1$, $k_{2}=20, x_{c}=0.3$, and for different values of the parameter $\gamma=10$, $20,100,500$, and 1000 , to be employed in the solution of Eq. (20). To the graph scale, the last two values $\gamma=500$ and 1000 produce a practically vertical variation on the thermophysical properties.

It should be recalled that there exists the exact solution for the linear heat conduction multiregion problem, with constant coefficients in each layer [10], but the aim here is not to solve the discontinuous problem, which would require a discontinuous eigenvalue problem to be formally correct [10,21], but a general version of the problem with variable properties, testing it in handling very sharp space variations on the coefficients. This is particularly important when dealing with thermophysical properties identification in such cases where interface positions between different materials are not known a priori and/or there is an actual transition region where it is crucial to estimate the properties variation.

Tables $2 \mathrm{a}$ and $\mathrm{b}$ illustrate the convergence behavior of the first 10 eigenvalues of the double-layer problem, Eq. (20), for the values

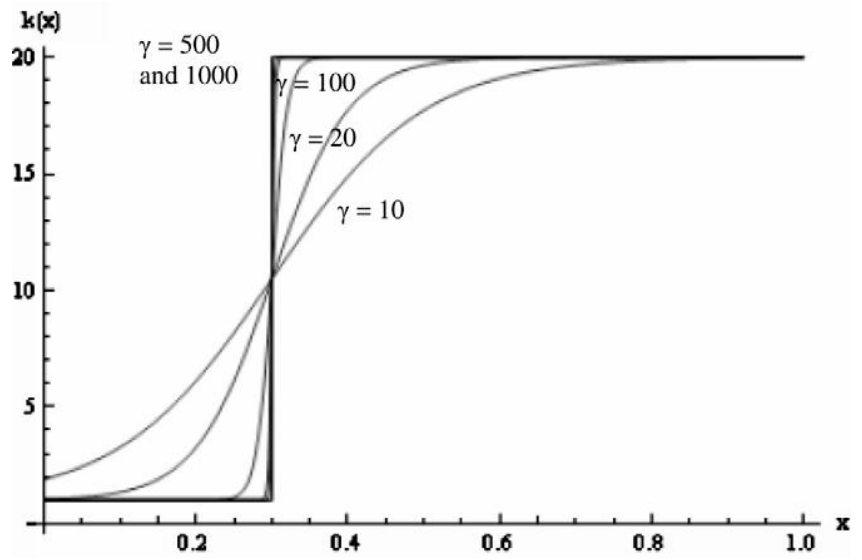

Fig. 3. Behavior of the variable diffusion coefficient $k(x)$ for the double-layer example, with $\gamma=10,20,100,500$, and 1000

Table 2a

Convergence of eigenvalues for double-layer problem, Eq. (20), with $\gamma=100$.

\begin{tabular}{lccccc}
\hline Eigenvalue $\mu_{i}$ & $N=30$ & $N=60$ & $N=90$ & $N=120$ & Discont. problem \\
\hline 1 & 5.69548 & 5.69249 & 5.69248 & 5.69248 & 5.21316 \\
2 & 10.0904 & 10.0903 & 10.0903 & 10.0903 & 10.0779 \\
3 & 16.9740 & 16.9645 & 16.9645 & 16.9645 & 15.6389 \\
4 & 20.2694 & 20.2674 & 20.2673 & 20.2673 & 20.1568 \\
5 & 27.9236 & 27.9082 & 27.9081 & 27.9081 & 26.0627 \\
6 & 30.6674 & 30.6564 & 30.6564 & 30.6564 & 30.2380 \\
7 & 38.4493 & 38.4303 & 38.4303 & 38.4303 & 36.4832 \\
8 & 41.3598 & 41.3228 & 41.3227 & 41.3227 & 40.3228 \\
9 & 48.6995 & 48.6742 & 48.6742 & 48.6742 & 46.8986 \\
10 & 52.2297 & 52.1382 & 52.1380 & 52.1380 & 50.4129 \\
\hline
\end{tabular}

Table 2b

Convergence of eigenvalues for double-layer problem, Eq. (20), with $\gamma=500$.

\begin{tabular}{lccccc}
\hline Eigenvalue $\mu_{i}$ & $N=30$ & $N=60$ & $N=90$ & $N=120$ & Discont. problem \\
\hline 1 & 5.38136 & 5.32149 & 5.30854 & 5.30481 & 5.21316 \\
2 & 10.0791 & 10.0785 & 10.0784 & 10.0784 & 10.0779 \\
3 & 16.1432 & 15.9615 & 15.9227 & 15.9115 & 15.6389 \\
4 & 20.1674 & 20.1623 & 20.1614 & 20.1612 & 20.1568 \\
5 & 26.9008 & 26.5920 & 26.5274 & 26.5090 & 26.0627 \\
6 & 30.2779 & 30.2577 & 30.2544 & 30.2535 & 30.2380 \\
7 & 37.6438 & 37.2041 & 37.1147 & 37.0893 & 36.4832 \\
8 & 40.4352 & 40.3739 & 40.3648 & 40.3623 & 40.3228 \\
9 & 48.3439 & 47.7844 & 47.6730 & 47.6416 & 46.8986 \\
10 & 50.6907 & 50.5246 & 50.5034 & 50.4977 & 50.4129 \\
\hline
\end{tabular}

$\gamma=100$ and 500, respectively, for increasing truncation orders in the eigenfunction expansions, $N=30,60,90,120$, with $k_{1}=1$, $k_{2}=20, x_{c}=0.3, w_{1}=1$ and $w_{2}=4$. Also, the last column illustrates the exact results for the discontinuous double-layer problem, just for reference purposes, but not as a benchmarking tool, since it cannot be formally recovered by the present approach with a continuous auxiliary problem. Here, the equation coefficients are again not yet expanded in eigenfunctions, but taken from the analytical expressions in Eq. (21).

The very first eigenvalue $\left(\mu_{0}=0\right)$ was omitted from the tables since it is exactly recovered in all cases. For the less abrupt situation with $\gamma=100$ (Table 2a, the first 10 eigenvalues are already fully converged to the sixth significant digit with truncation orders of $N=90$ or smaller, while for the very sharp interface with $\gamma=500$ (Table 2b), $N=120$ terms are required to achieve three to four significant digits of convergence in the eigenvalues. One may also observe the tendency of the eigenvalues towards the two-region results as the transition region between the two layers is made narrower. 
Fig. 4 presents the behavior of the 10th eigenfunction for the most abrupt case of $\gamma=1000$, for different truncation orders, $N=30,60,90$, and 120 , with different symbols, together with the double-layer exact results, in solid line. To the graph scale, the eigenfunction is already converged to $N=60$ terms, with a noticeable slightly better convergence behavior in the first region $\left(x<x_{c}\right)$.

Also, Fig. 5 illustrates the excellent convergence behavior of the expansions for the temperature distribution in this double-layer example, again in the most abrupt case $(\gamma=1000)$, at three different values of the time variable, $t=0.001,0.01$, and 0.05 . One may notice a practically full coincidence of all curves to the graph scale within this range of the truncation orders. It is also observable that the results offer a very good reproduction of the exact discontinuous interface two-layer results.

Table 3 below further demonstrates this excellent convergence behavior for the most abrupt case $(\gamma=1000)$, by presenting the temperature values at the edge of the second layer, $x=1$, where the convergence of the eigenfunctions is apparently slower, for time $t=0.01$. The same truncation orders are considered, $N=30$, 60,90 , and 120 in terms of the eigenfunctions of the auxiliary problem, and up to the 11th eigenvalue of the original heterogeneous problem. Clearly, the temperature values are fully converged to the sixth digit up to order $i=6$ of the eigenfunction expansion of

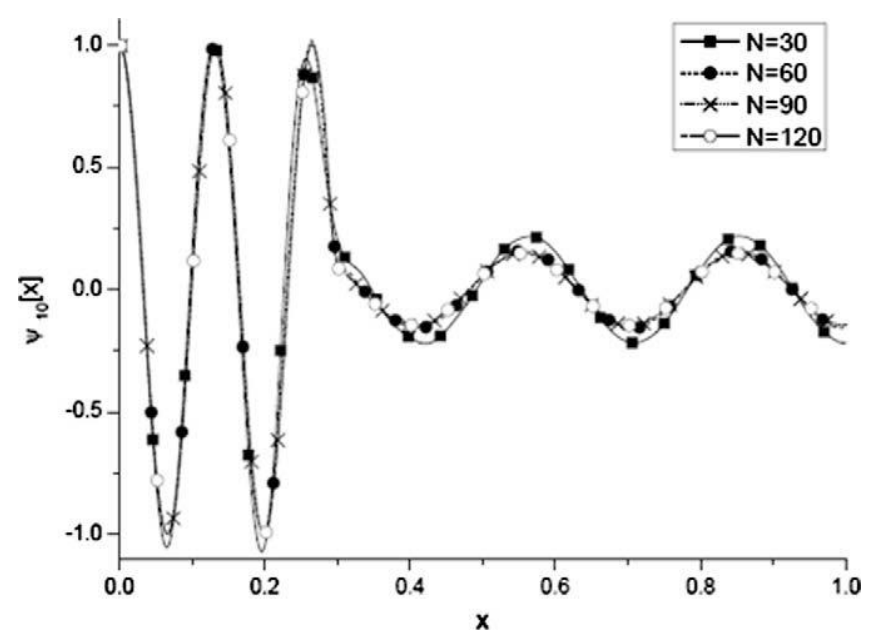

Fig. 4. Convergence behavior of the 10th eigenfunction $\psi_{10}(x)$ for the double-layer example, with $\gamma=1000$, and truncation orders $N=30,60,90$, and 120 .

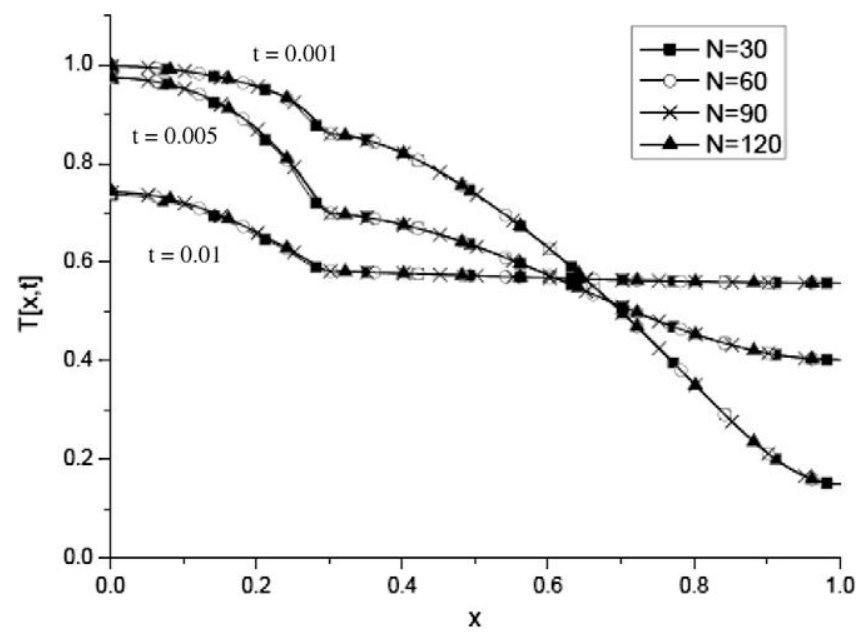

Fig. 5. Convergence behavior of the temperature profile for the double-layer example, with $\gamma=1000$, and truncation orders $N=30,60,90$, and 120 .
Table 3

Convergence of temperature distribution for double-layer problem, Eq. (20), with $\gamma=1000$.

\begin{tabular}{llllll}
\hline Order $i$ & $N=30$ & $N=60$ & $N=90$ & $N=120$ & Discont. problem \\
\hline 0 & 0.578603 & 0.578603 & 0.578603 & 0.578603 & 0.578602 \\
1 & 0.521377 & 0.521690 & 0.521724 & 0.521768 & 0.521924 \\
2 & 0.403499 & 0.403586 & 0.403567 & 0.403588 & 0.403616 \\
3 & 0.402522 & 0.402532 & 0.402491 & 0.402500 & 0.402461 \\
4 & 0.401761 & 0.401764 & 0.401721 & 0.401730 & 0.401686 \\
5 & 0.401756 & 0.401758 & 0.401716 & 0.401724 & 0.401681 \\
6 & 0.401752 & 0.401755 & 0.401712 & 0.401721 & 0.401677 \\
7 & 0.401752 & 0.401755 & 0.401712 & 0.401721 & 0.401677 \\
8 & 0.401752 & 0.401755 & 0.401712 & 0.401721 & 0.401677 \\
9 & 0.401752 & 0.401755 & 0.401712 & 0.401721 & 0.401677 \\
10 & 0.401752 & 0.401755 & 0.401712 & 0.401721 & 0.401677 \\
\hline
\end{tabular}

the original problem, in all cases, including the exact solution for the discontinuous double-layer. In the other sense, of increasing $N$, one may see that the temperature field is converged to at least four significant digits, even for $N=30$, and agreeing with the discontinuous problem exact solution also in four significant digits.

Next, we illustrate the convergence of the eigenfunction expansion representations of the variable coefficients themselves, in both cases of a double layer with a transition region and the randomly variable properties. For instance, Fig. $6 a$ and b illustrate
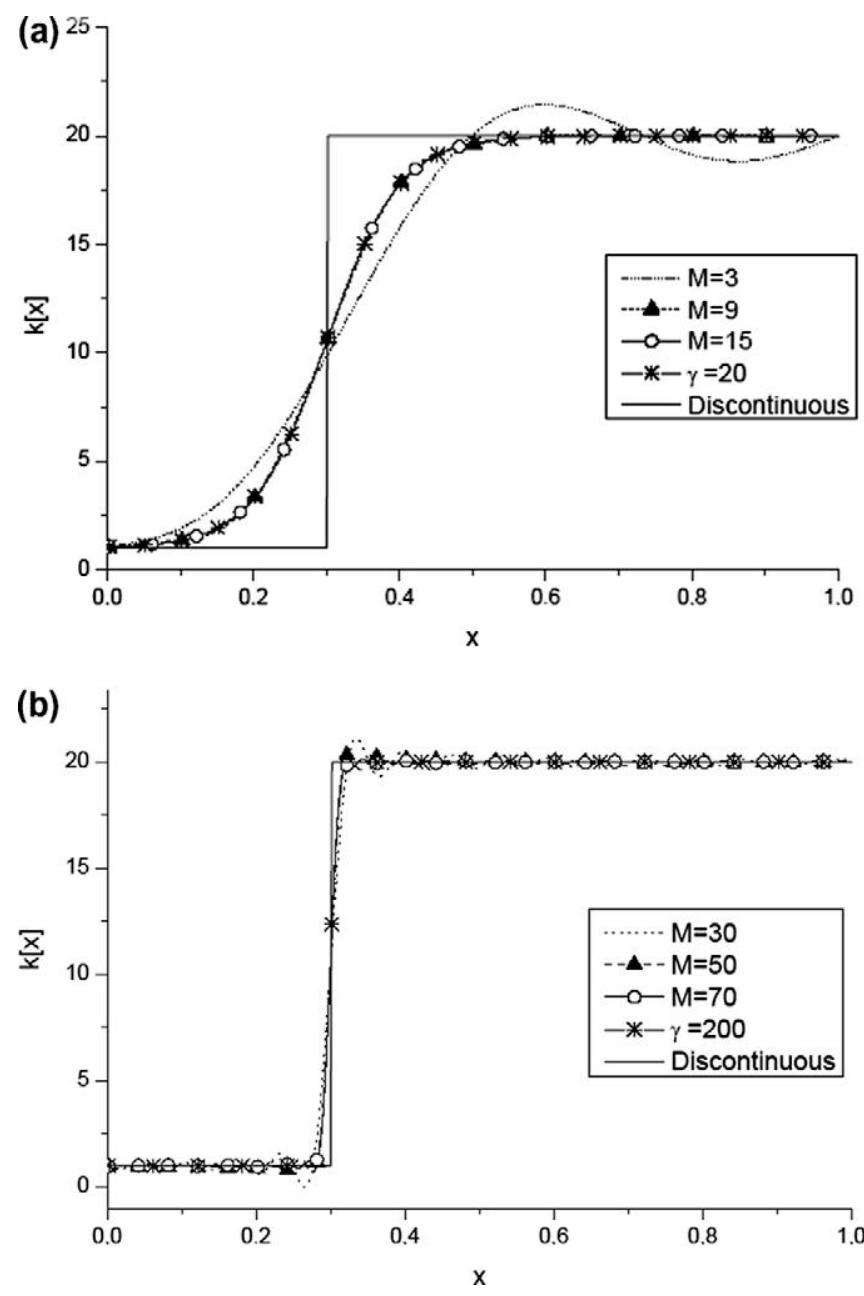

Fig. 6. (a) Behavior of the variable diffusion coefficient $k(x)$ and its eigenfunction expansion for the double-layer example, with $\gamma=20$ and truncation orders $M=3,9$, and 15.6. (b) Behavior of the variable diffusion coefficient $k(x)$ and its eigenfunction expansion for the double-layer example, with $\gamma=200$ and truncation orders $M=30$, 50 , and 70 . 
the behavior of the variable coefficient $k(x)$ for the double-layer example, together with its eigenfunction expansion, with $k_{1}=1$, $k_{2}=20, x_{c}=0.3$, and for different values of the parameter $\gamma=20$ and 200 . To the graph scale, the value $\gamma=200$ produces a practically vertical variation on the thermophysical properties. We have chosen to adopt as a filter for the coefficients expansion, the straight line that connects the two extreme values, $k(0)$ and $k(1)$, not accounting for the knowledge of the interface position. The eigenfunction was taken from the same auxiliary problem equation, but with first kind boundary conditions, i.e.:

$\tilde{\Gamma}_{k}(x)=\sqrt{2} \sin \left(v_{k} x\right), \quad$ with $v_{k}=k \pi, \quad k=1,2,3 \ldots$

For the case with the less abrupt space variation in the double layer example, Fig. 6a, convergence of the coefficient expansion is achieved to the graph scale with very low truncation orders, such as $M=6$ and $M=9$, which practically overwrite the curve for the original coefficient $k(x)$, Eq. (21a). For the case of an actual abrupt variation, Fig. 6b, a larger number of terms were required for the expansion to appropriately recover the coefficient behavior, as illustrated by the curve with $M=70$, which is practically coincident with the exact coefficient curve.

Similar results were obtained and analyzed for the random properties example, as illustrated below in Fig. 7a and b for the
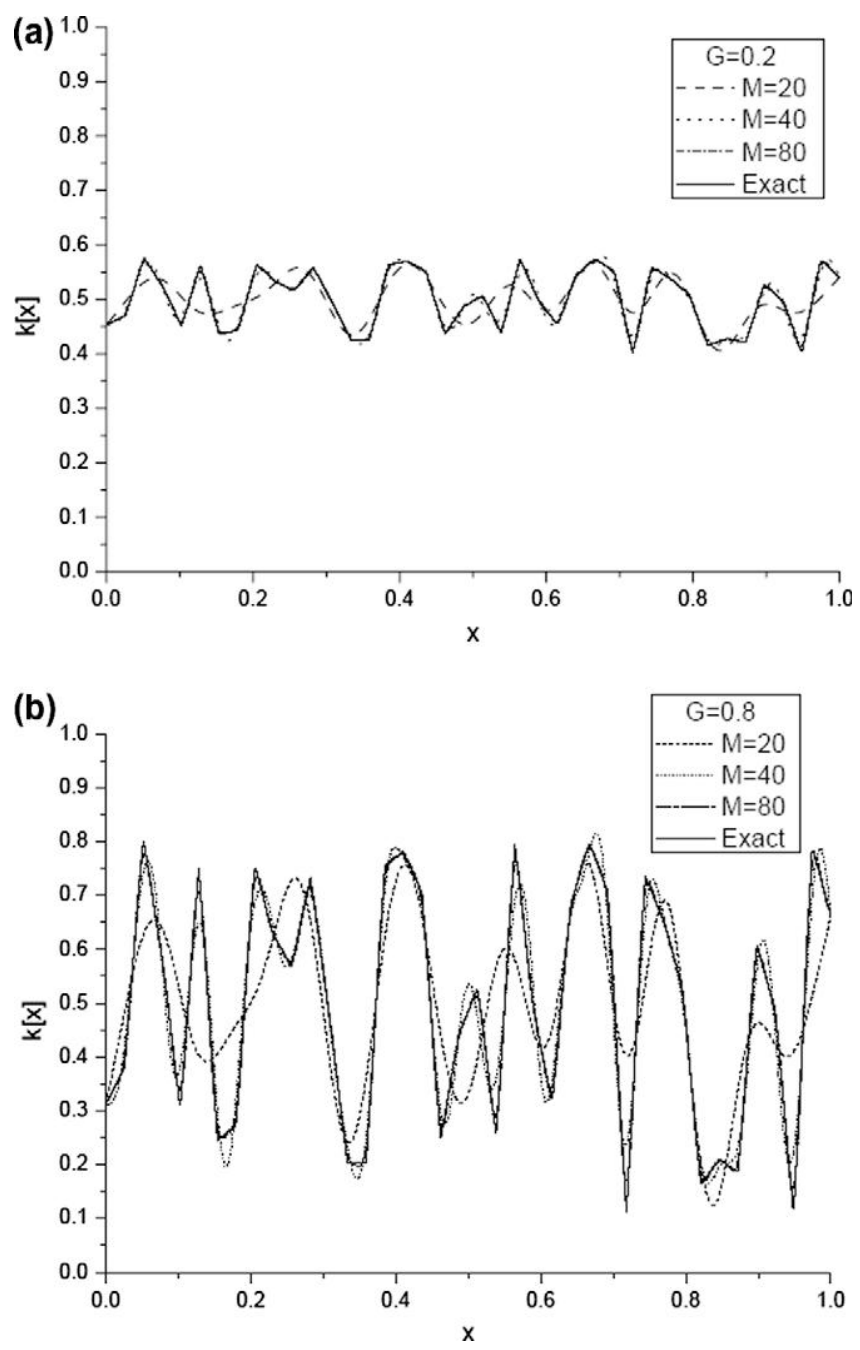

Fig. 7. (a) Behavior of the variable diffusion coefficient $k(x)$ and its eigenfunction expansion for the random properties example, with $G=0.2$ and truncation orders $M=20,40$, and 80 . (b) Behavior of the variable diffusion coefficient $k(x)$ and its eigenfunction expansion for the random properties example, with $G=0.8$ and truncation orders $M=20,40$, and 80 . $k(x)$ coefficient by taking $k_{0}=0.5$, with $G=0.2$ and 0.8 , respectively. A total of 40 equally spaced points were taken along the domain for the random properties generation, while the random numbers at each position were kept unchanged for the two cases of different gains. The truncation orders for the coefficient eigenfunction expansion are illustrated for $M=20,40$, and 80 . Clearly, the case with the smaller gain, $G=0.2$, presents a better convergence behavior, due to the dumping effect on the oscillations amplitudes, with the results for $M=80$ being fully coincident with the interpolated original curves that are overwritten by the expansion results. For the case with larger amplitudes in the random variations, $G=0.8$, the curve for $M=40$ still presents noticeable deviations from the original interpolated curve, while the curve for $M=80$ practically overwrites the original coefficient graph, except at the very sharp edges which could still require a few extra terms. The same trends were observed for the randomly generated $w(x)$ behavior, generated for $w_{0}=0.5$ and also for 40 equally spaced points, as illustrated in Fig. 8a and b below. As opposed to the case in [1], the two coefficients were allowed to be independently generated, to further challenge the proposed approach.

The eigenvalue problem solution obtained with the expanded equation coefficients is now demonstrated, first by considering the double-layer example, again with $k_{1}=1, k_{2}=20, x_{c}=0.3$,
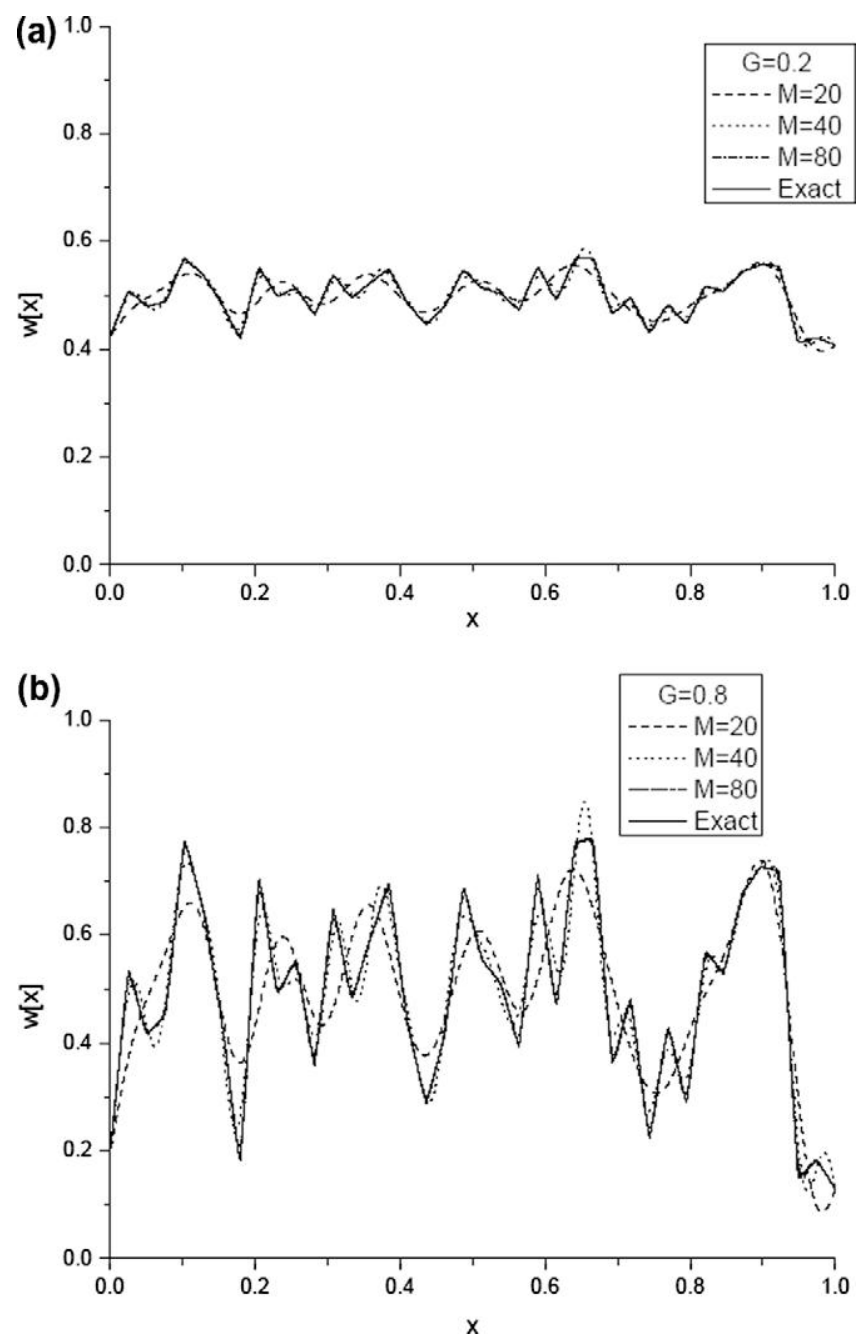

Fig. 8. (a) Behavior of the variable diffusion coefficient $w(x)$ and its eigenfunction expansion for the random properties example, with $G=0.2$ and truncation orders $M=20,40$, and 80 . (b) Behavior of the variable diffusion coefficient $\mathrm{w}(x)$ and its eigenfunction expansion for the random properties example, with $G=0.8$ and truncation orders $M=20,40$, and 80 . 
$w_{1}=1$, and $w_{2}=4$, with $\gamma=20$ and 200. Tables $4 \mathrm{a}$ and $\mathrm{b}$ then show the converged values of the first 10 eigenvalues $\mu_{i}$ 's for different truncation orders in the coefficients expansions, $M$, compared in the last two columns with the original continuous coefficient solution and the exact discontinuous eigenvalue problem solution, here shown just as a limiting case [26]. The eigenfunction expansions of the original eigenvalue problem itself were fixed to truncation orders $N=50$ in the case of $\gamma=20$, and $N=100$ for $\gamma=200$, which are more than sufficient to provide converged results to the first 10 eigenvalues here presented, as previously achieved for the original coefficients representation. In Table $4 a$, for the smoother coefficients behavior, fairly low truncation orders $(M=27)$ in the coefficients expansions already provide four significant digits of convergence in the first 10 eigenvalues, as compared to the eigenvalues obtained with the numerical integration based on the original coefficients representations. On the other hand, for the very abrupt variation case, for $\gamma=200$, it is shown in Table $4 \mathrm{~b}$ that $M=110$ terms are required to yield four fully converged significant digits in these same first 10 eigenvalues. Again, it is clear that the results in Table $4 \mathrm{~b}$ are closer to the exact solution of the discontinuous case, as the coefficients representation approach constant values in each layer, in contrast with the case of $\gamma=20$.

Now, the random properties case is more closely examined, initiating by the illustration of the convergence behavior of the first 10 eigenvalues for a fixed order in the coefficients expansion $(M=60)$ but with increasing order in the eigenfunction expansion of the original problem $(N<150)$. The aim is to demonstrate that the proposed approach is capable of reaching convergence on the eigenvalues of such a variable coefficients behavior for the worst case of $G=1$, to within reasonable values of the expansion orders. As can be observed in Table 5 below, at least four significant digits are fully converged within the first 10 eigenvalues, in the present range of truncation orders for the original problem eigenfunction expansion $(N)$.

In addition, the influence of the coefficients expansions truncation orders $(M)$ on the behavior of the eigenvalues of problem (20) is investigated, for the selected truncation orders, $M=20,40,60$, and 80, and the coefficients given as in Figs. 7 and $8 \mathrm{a}$ and b, respectively for $G=0.2$ and 0.8 . The fully converged first 10 eigenvalues are shown for the four truncation orders in Tables $6 a$ and b, while the last column stands for the exact solution of the constant properties case taking the average values $\left(k_{0}=0.5, w_{0}=0.5\right)$, which corresponds to letting $G=0$. One may see that the case $G=0.2$ (Table $6 a)$ presents a faster convergence behavior, with five significant digits being fully converged at $M=80$, and four digits even at much lower orders $(M=40)$. The case $G=0.8$ (Table 6 b) requires $M=80$ for convergence to three or four digits Also, the results for the case $G=0.2$ are much closer to those of the average coefficients case than for the case of larger amplitudes $(G=0.8)$.

Table 4a

Influence of the coefficients expansion order on the eigenvalues for double-layer problem and $\gamma=20$.

\begin{tabular}{|c|c|c|c|c|c|c|c|}
\hline Eigenvalue $\mu_{i}$ & $M=3$ & $M=9$ & $M=15$ & $M=21$ & $M=27$ & Original coefficients & Discont. problem \\
\hline 1 & 7.86584 & 7.56036 & 7.58226 & 7.58278 & 7.58282 & 7.58283 & 5.21316 \\
\hline 2 & 12.0937 & 11.0779 & 11.1062 & 11.1072 & 11.1073 & 11.1073 & 10.0779 \\
\hline 3 & 18.1562 & 18.1547 & 18.1168 & 18.1191 & 18.1192 & 18.1192 & 15.6389 \\
\hline 4 & 24.1409 & 23.6115 & 23.4188 & 23.4229 & 23.4232 & 23.4233 & 20.1568 \\
\hline 5 & 30.0858 & 29.2754 & 29.1625 & 29.1583 & 29.1588 & 29.1589 & 26.0627 \\
\hline 6 & 36.0473 & 35.0508 & 35.0189 & 35.0032 & 35.0038 & 35.0040 & 30.238 \\
\hline 7 & 42.0149 & 40.8188 & 40.7035 & 40.6934 & 40.6930 & 40.6934 & 36.4832 \\
\hline 8 & 47.9863 & 46.6067 & 46.5132 & 46.5116 & 46.5098 & 46.5102 & 40.3228 \\
\hline 9 & 53.9608 & 52.3996 & 52.2864 & 52.2758 & 52.2743 & 52.2744 & 46.8986 \\
\hline 10 & 59.9374 & 58.1925 & 58.0674 & 58.0627 & 58.0623 & 58.0618 & 50.4129 \\
\hline
\end{tabular}

Table $4 b$

Influence of the coefficients expansion order on the eigenvalues for double-layer problem and $\gamma=200$.

\begin{tabular}{|c|c|c|c|c|c|c|c|}
\hline Eigenvalue $\mu_{i}$ & $M=30$ & $M=50$ & $M=70$ & $M=90$ & $M=110$ & Original coefficients & Discont. problem \\
\hline 1 & 3.92070 & 5.35783 & 5.43630 & 5.44375 & 5.44380 & 5.44376 & 5.21316 \\
\hline 2 & 10.0070 & 10.0756 & 10.0800 & 10.0805 & 10.0805 & 10.0805 & 10.0779 \\
\hline 3 & 13.5127 & 16.0994 & 16.2970 & 16.3147 & 16.3140 & 16.3139 & 15.6389 \\
\hline 4 & 19.9172 & 20.1566 & 20.1772 & 20.1793 & 20.1794 & 20.1794 & 20.1568 \\
\hline 5 & 24.3117 & 26.8147 & 27.1049 & 27.1299 & 27.1286 & 27.1284 & 26.0627 \\
\hline 6 & 29.7991 & 30.2606 & 30.3167 & 30.3214 & 30.3213 & 30.3213 & 30.238 \\
\hline 7 & 35.5958 & 37.4502 & 37.8105 & 37.8416 & 37.8407 & 37.8402 & 36.4832 \\
\hline 8 & 39.6450 & 40.4020 & 40.5349 & 40.5439 & 40.5431 & 40.5431 & 40.3228 \\
\hline 9 & 49.1208 & 47.9772 & 48.3633 & 48.3998 & 48.4000 & 48.3994 & 46.8986 \\
\hline 10 & 49.7108 & 50.5804 & 50.8729 & 50.8906 & 50.8884 & 50.8882 & 50.4129 \\
\hline
\end{tabular}

Table 5

Convergence of the first 10 eigenvalues for the random properties case, with $G=1$, and $M=60$.

\begin{tabular}{|c|c|c|c|c|c|c|c|}
\hline Eigenvalue $\mu_{i}$ & $N=30$ & $N=50$ & $N=70$ & $N=90$ & $N=110$ & $N=130$ & $N=150$ \\
\hline 1 & 2.90236 & 2.81658 & 2.79154 & 2.78586 & 2.78378 & 2.78283 & 2.78251 \\
\hline 2 & 5.23446 & 5.10402 & 5.02386 & 5.00907 & 5.00218 & 4.99923 & 4.99836 \\
\hline 3 & 8.10146 & 7.98014 & 7.92516 & 7.91856 & 7.91641 & 7.91562 & 7.91551 \\
\hline 4 & 11.0150 & 10.7348 & 10.6866 & 10.6733 & 10.6694 & 10.6673 & 10.6667 \\
\hline 5 & 14.2056 & 13.7055 & 13.5243 & 13.4471 & 13.4149 & 13.4000 & 13.3948 \\
\hline 6 & 18.0474 & 17.5089 & 17.4113 & 17.3497 & 17.3335 & 17.3257 & 17.3232 \\
\hline 7 & 21.7988 & 21.3903 & 21.3198 & 21.2922 & 21.2888 & 21.2873 & 21.2869 \\
\hline 8 & 23.8719 & 22.7118 & 22.4950 & 22.3415 & 22.3052 & 22.2910 & 22.2843 \\
\hline 9 & 26.1803 & 25.4689 & 25.2843 & 25.2324 & 25.2139 & 25.2064 & 25.2034 \\
\hline 10 & 28.0210 & 27.1340 & 26.9713 & 26.9298 & 26.9163 & 26.9121 & 26.9104 \\
\hline
\end{tabular}


Table 6a

Influence of the coefficients expansion order on the eigenvalues convergence for random properties case, with $G=0.2$, and $N=130$.

\begin{tabular}{lccccc}
\hline Eigenvalue $\mu_{i}$ & $M=20$ & $M=40$ & $M=60$ & $M=80$ & $\begin{array}{l}\text { Average } \\
\text { coefficients }\end{array}$ \\
\hline 1 & 3.16555 & 3.15678 & 3.15695 & 3.15686 & 3.14159 \\
2 & 6.28652 & 6.26833 & 6.26858 & 6.26838 & 6.28319 \\
3 & 9.36202 & 9.33868 & 9.33895 & 9.33878 & 9.42478 \\
4 & 12.6601 & 12.6152 & 12.6163 & 12.6160 & 12.5664 \\
5 & 15.7812 & 15.7351 & 15.7352 & 15.7347 & 15.7080 \\
6 & 19.0825 & 19.0163 & 19.0168 & 19.0160 & 18.8496 \\
7 & 22.2211 & 22.1142 & 22.1176 & 22.1168 & 21.9911 \\
8 & 25.0214 & 24.8849 & 24.8881 & 24.8868 & 25.1327 \\
9 & 28.1124 & 27.9357 & 27.9379 & 27.9368 & 28.2743 \\
10 & 31.4528 & 31.0315 & 31.0302 & 31.0296 & 31.4159 \\
\hline
\end{tabular}

\section{Table 6b}

Influence of the coefficients expansion order on the eigenvalues convergence for random properties case, with $G=0.8$, and $N=130$.

\begin{tabular}{lccccc}
\hline Eigenvalue $\mu_{i}$ & $M=20$ & $M=40$ & $M=60$ & $M=80$ & $\begin{array}{l}\text { Average } \\
\text { coefficients }\end{array}$ \\
\hline 1 & 3.09140 & 2.99458 & 2.98961 & 2.98921 & 3.14159 \\
2 & 5.81296 & 5.67551 & 5.65526 & 5.65416 & 6.28319 \\
3 & 8.69607 & 8.50171 & 8.50780 & 8.51193 & 9.42478 \\
4 & 12.2436 & 11.6288 & 11.6549 & 11.6589 & 12.5664 \\
5 & 15.2666 & 14.8153 & 14.7007 & 14.6916 & 15.7080 \\
6 & 19.1082 & 18.4638 & 18.3620 & 18.3454 & 18.8496 \\
7 & 22.9467 & 21.7388 & 21.7486 & 21.7476 & 21.9911 \\
8 & 24.9176 & 24.0072 & 23.9370 & 23.8987 & 25.1327 \\
9 & 27.6446 & 26.3021 & 26.3031 & 26.2804 & 28.2743 \\
10 & 31.4090 & 28.3980 & 28.4054 & 28.4077 & 31.4159 \\
\hline
\end{tabular}

Finally, we examine the behavior of the temperature distribution within the random properties medium, as a function of the gain $G$ for the values $G=0,0.2,0.5,0.8$, and 1 , which governs the amplitude of the coefficients variations, but maintaining the same random numbers at each $x$ for the different gains. Fig. 9a and b illustrate the temperature profile behavior at two different dimensionless times, respectively, for $t=0.05$ and 0.1 . The base case $G=0$ provides the result for the constant properties situation, when the properties local variations are ignored and substituted by an effective average value. As we can see, the differences between the variable and constant coefficients cases are more significant for increasing $G$ and time value, and closer to the boundary $x=1$ for this particular application. A reasonable reproduction of the actual heterogeneous problem solution when employing effective values, is achieved only for the moderate case of $G=0.2$.

\section{Conclusions}

The Generalized Integral Transform Technique (GITT) is employed in the analytical solution of eigenvalue problems related to diffusion in heterogeneous media, represented by arbitrarily space variable equation coefficients. The handling of such SturmLiouville problems in terms of eigenfunction expansions of simpler problems of known solution, allows for the straightforward application of well established exact solutions for transient diffusion in heterogeneous media.

Three quite different situations were here considered to challenge the proposed approach, namely, the case of functionally graded materials with properties varying across the space coordinate within orders of magnitude, the case of a double layer with a sudden transition region, and the situation of dispersed systems with randomly variable concentrations and thus physical properties. Convergence behavior was extensively illustrated for both eigenvalues and eigenfunctions, as well as for the temperature dis-
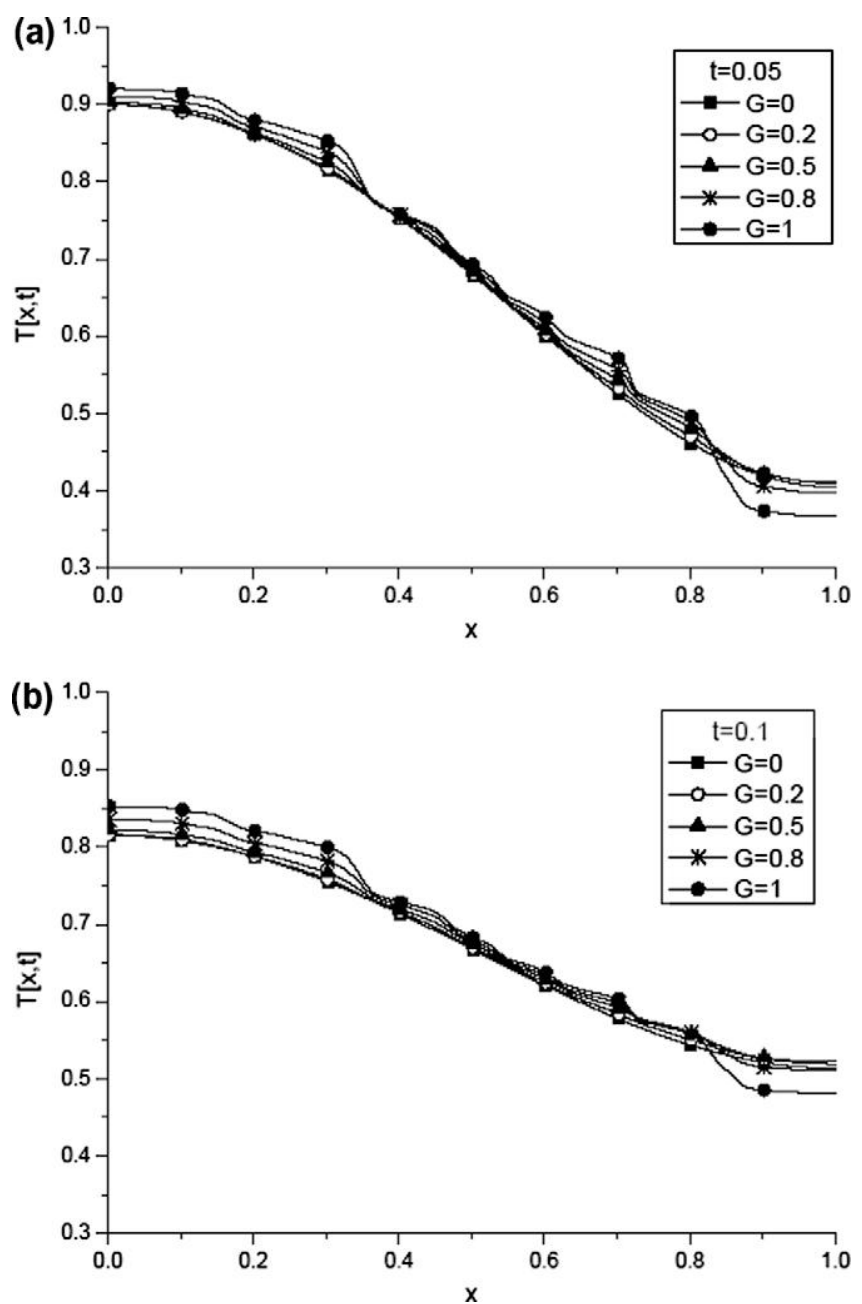

Fig. 9. (a) Temperature distribution for the random properties medium at $t=0.05$, with $G=0,0.2,0.5,0.8$, and 1 , and truncation orders $N=130$ and $M=80$. (b) Temperature distribution for the random properties medium at $t=0.1$, with $G=0$, $0.2,0.5,0.8$, and 1 , and truncation orders $N=130$ and $M=80$.

tributions of the originally posed problems. Though the solution for the sharp variation of properties closely reproduces the actually discontinuous problem results, in order to formally handle a discontinuous original eigenvalue problem, the auxiliary problem needs to be chosen itself discontinuous for an exact satisfaction of the continuity relations, such as in [21]. In this case, the formal solution above presented is essentially the same and directly applicable. Also, the numerical examples presented were chosen to be one-dimensional in order to illustrate the three different possible natures of the properties variations, to within the available space, but the formal solution and computational algorithm are easily extended to two- or three-dimensional situations provided one accounts for the adequate reordering of terms in the eigenfunction expansions, such as in [24,27].

Perhaps the major novelty with respect to the previous implementations of the integral transform method here reviewed, lies on that the present methodology introduces the idea of expanding the variable coefficients themselves in terms of known eigenfunctions, so as to allow for the full analytical implementation of the approach during assembly of the coefficients matrices required in the transformed eigenvalue problem formulation. Such modification opens up new perspectives in the utilization of the methodology in either physical properties mapping in connection with inverse problems analysis and/or materials properties tailoring and optimization tasks. 


\section{Acknowledgements}

The authors acknowledge the financial support provided by CNPq, Brasil, and CNRS, France. This paper is dedicated to the memory of Prof. M. Necati Ozisik, prominent scientist and intellectual fortress for the advancement of analytical heat and mass transfer.

\section{References}

[1] S.H. Lin, Transient conduction in heterogeneous media, Int. Comm. Heat Mass Transfer 10 (1992) 165-174.

[2] F. Qiulin, X. Xingcheng, H. Xingfang, G. Jingkun, Calculating method of the equivalent thermal conductivity of functionally gradient materials, Mater. Sci. Eng. A261 (1999) 84-88.

[3] F. Danes, B. Garnier, T. Dupuis, Predicting measuring and tailoring the transverse thermal conductivity of composites from polymer matrix and metal filler, Int. J. Thermophys. 24 (2003) 771-784.

[4] I.H. Tavman, H. Akinci, Transverse thermal conductivity o fiber reinforced polymer composites, Int. Comm. Heat Mass Transfer 27 (2000) 253-261.

[5] E. Divo, A. Kassab, Generalized boundary integral equation for transient heat conduction in heterogeneous media, J. Thermophys. Heat Transfer 12 (1998) 364

[6] O. Fudym, B. Ladevie, J.C. Batsale, A seminumerical approach for heat diffusion in heterogeneous media: one extension of the analytical quadrupole method, Num. Heat Transfer B: Fundam. 42 (2002) 325-348.

[7] A. Sutradhar, G.H. Paulino, L.J. Gray, Transient heat conduction in homogeneous and non-homogeneous materials by the Laplace Transform Galerkin Boundary Element Method, Eng. Anal. Boundary Elem. 26 (2002) 119-132.

[8] F. Jiang, A.C.M. Sousa, Effective thermal conductivity of heterogeneous multicomponent materials: an SPH implementation, Heat Mass Transfer 43 (2007) 479-491.

[9] Y. Dai, W. Tan, Q. Sun, Y.D. Li, Effect of different thermal conductivity functions on temperature fields in FGM, J. Mater. Process. Technol. 187-188 (2007) 212214.

[10] M.D. Mikhailov, M.N. Ozisik, Unified Analysis and Solutions of Heat and Mass Diffusion, John Wiley, New York, 1984.

[11] R.M. Cotta, Hybrid numerical-analytical approach to nonlinear diffusion problems, Num. Heat Transfer B: Fundam. 127 (1990) 217-226.
[12] R.M. Cotta, Integral Transforms in Computational Heat and Fluid Flow, CRC Press, Boca Raton, Florida, 1993.

[13] R.M. Cotta, Benchmark results in computational heat and fluid flow: - the integral transform method, Int. J. Heat Mass Transfer 37 (Suppl. 1) (1994) 381394 (invited paper).

[14] R.M. Cotta, M.D. Mikhailov, Heat Conduction: Lumped Analysis Integral Transforms Symbolic Computation, Wiley Interscience, New York, 1997.

[15] R.M. Cotta (Ed.), The Integral Transform Method in Thermal and Fluids Sciences and Engineering, Begell House, New York, 1998.

[16] R.M. Cotta, M.D. Mikhailov, Hybrid methods and symbolic computations, in: W.J. Minkowycz, E.M. Sparrow, J.Y. Murthy (Eds.), Handbook of Numerical Heat Transfer, second ed., John Wiley, New York, 2006, pp. 493-522.

[17] S. Wolfram, The Mathematica Book, version 5.2, Cambridge-Wolfram Media, 2005.

[18] P.B. Bailey, M.K. Gordon, L.F. Shampine, Automatic solution of the SturmLiouville problem, ACM Trans. Math. Software 4 (1978) 193-208.

[19] P.B. Bailey, B.S. Garbow, H.G. Kaper, A. Zettl, Eigenvalue and eigenfunction computations for Sturm-Liouville problems, ACM Trans. Math. Software 17 (1991) 491-499.

[20] M.D. Mikhailov, N.L. Vulchanov, A computational procedure for SturmLiouville problems, J. Comput. Phys. 50 (1983) 323-336.

[21] R.M. Cotta, E. Nogueira, On the eigenvalues basic to diffusion through composite media, Comput. Appl. Math. 7 (1988) 201-213.

[22] M.D. Mikhailov, R.M. Cotta, Integral transform method for eigenvalue problems, Comm. Num. Meth. Eng. 10 (1994) 827-835.

[23] M.C. Oliveira, R. Ramos, R.M. Cotta, On the eigenvalues basic to the analytical solution of convective heat transfer with axial diffusion effects, Comm. Num. Meth. Eng. 11 (1995) 287-296.

[24] L.A. Sphaier, R.M. Cotta, Integral transform analysis of multidimensional eigenvalue problems within irregular domains, Num. Heat Transfer B: Fundam. 38 (2000) 157-175.

[25] O. Fudym, H.R.B. Orlande, M. Bamford, J.C. Batsale, Bayesian approach for thermal diffusivity mapping from infrared images processing with spatially random heat pulse heating, in: Sixth ICIPE Proceedings, Sixth International Conference on Inverse Problems in Engineering: Theory and Practice, Dourdan, France, June 15th-19th, 2008.

[26] C.P. Naveira, O. Fudym, R.M. Cotta, H.R.B. Orlande, Integral transform solutions for diffusion in heterogeneous media, in: Proceedings of IMECE2008, ASME International Mechanical Engineering Congress \& Exposition, Paper No. IMECE2008-69114, Boston, MA, November 2008.

[27] E.J. Correa, R.M. Cotta, H.R.B. Orlande, On the reduction of computational costs in eigenfunction expansions of multidimensional diffusion problems, Int. J. Num. Meth. Heat Fluid Flow 7 (1997) 675-695. 